\title{
Comment on: The joy of PARENTING: INFANT SLEEP INTERVENTION TO IMPROVE MATERNAL EMOTIONAL
} WELL-BEING AND INFANT SLEEP

Singapore Med J 2017; 58(3): 167 doi: 10.11622/smedj.2017019

Dear Sir,

I read with interest the recent article by Symon and Crichton $^{(1)}$ on infant sleep interventions. For such an important topic, there are two main issues arising from this article.

Firstly, the intervention utilised is lacking in detail, making it difficult to interpret the findings. The article reports the use of behavioural sleep interventions in infants aged 6-12 months, using the same protocol the authors have published elsewhere. ${ }^{(2)}$ The protocol describes the use of extinction techniques as a sleep promotion strategy; these are based on extinction theory, which proposes that a behavioural response (crying) is maintained or strengthened by the presence of a reinforcer (parental attention). ${ }^{(3)}$ Extinction sleep interventions aim to remove parental attention to the child during sleep periods so that infants can 'learn' to self-settle without parental assistance. ${ }^{(4)}$ As in their original protocol, it appears that Symon and Crichton instructed parents to regard "crying at sleep initiation as being due to tiredness and best handled by minimal parental response ${ }^{\prime \prime} .{ }^{(2)}$ This statement is not based on evidence and my concern is that it may even be misinformation, given that it is impossible to ascertain whether crying is due to tiredness or perhaps something else, such as separation from the parent. Indeed, the authors describe crying as "inappropriate behaviour". The authors have entirely omitted to describe in detail how they should differentiate between cries resulting from pain and those resulting from separation. Furthermore, the described intervention assumes that the infant is not distressed in any way, and that the crying has desisted not from learned helplessness, but from acceptance. These simplistic assumptions cannot be verified.

Secondly, the article does not discuss why 21 families out of 80 (26\% attrition rate) did not complete the study, with only 49 returning $(61 \%)$ for the follow-up interview. Empirical studies normally report reasons for attrition, as it may result in attrition bias. The sample in this study already has a self-selection bias. Although we are not told, it is entirely possible that those 21 participants withdrew due to difficulty with extinction-based methods. Several reviews over many years cite studies in which parental resistance is one of the main drivers of attrition due to difficulties in compliance with extinction interventions. ${ }^{(5-8)}$ Extinction theory is clear that parents are only successful when they ignore their distressed and crying child. ${ }^{(4)}$ Studies of extinction-based interventions have noted attrition rates of $10 \%-30 \%$, with parents resisting extinction interventions because they are too traumatic, contradict their beliefs about child-rearing or are impractical. ${ }^{(4)}$ In anonymous online surveys reported in Blunden et al, $60 \%-70 \%$ of parents reported that they would not use or stopped using extinction-based sleep interventions, citing emotional distress. ${ }^{(4)}$

Parents of sleep-disturbed infants are very vulnerable and at risk. Non-extinction-based interventions are available and general practitioners, who are the primary point of call for families in need, must receive evidence-based information that is not selective but comprehensive in reporting research findings.

Yours sincerely,

Sarah Blunden ${ }^{1}$

${ }^{1}$ Paediatric Sleep Research, CQUniversity, Appleton Institute, South Australia, Australia. s.blunden@cqu.edu.au

\section{REFERENCES}

1. Symond B, Crichton GE. The joy of parenting: infant sleep intervention to improve maternal emotional well-being and infant sleep. Singapore Med J 2016; 58:50-4.

2. Symon B, Marley JE, Martin AJ, Norma ER. Effect of a consultation teaching behaviour modification on sleep performance in infants: a randomised controlled trial. Med J Aust 2005; 182:215-8.

3. Bouton ME. Context and behavioral processes in extinction. Learn Mem 2004; 11:485-94.

4. Blunden S, Etherton H, Hauck Y. Resistance to cry intensive sleep intervention in young children: are we ignoring children's cries or parental concerns? Children 2016; 3:pii E8.

5. Mindell JA, Kuhn B, Lewin DS, Meltzer LJ, Sadeh A; American Academy of Sleep Medicine. Behavioral treatment of bedtime problems and night wakings in infants and young children. Sleep 2006; 29:1263-76.

6. Meltzer LJ. Clinical management of behavioral insomnia of childhood: treatment of bedtime problems and night wakings in young children. Behav Sleep Med 2010; 8:172-89.

7. Ramos KD, Youngclarke DM. Parenting advice books about child sleep: cosleeping and crying it out. Sleep 2006; 29:1616-23.

8. Blunden SL, Thompson KR, Dawson D. Behavioural sleep treatments and night time crying in infants: challenging the status quo. Sleep Med Rev 2011; 15:327-34. 